\title{
Análisis bromatológico de la leche a partir de la semilla de alpiste (phalaris canarienses): cereal empleado como sustituto dietético
}

\section{Bromatological analysis of milk from canary seed (phalaris canarienses): cereal used as a dietary substitute}

\author{
Peña C. Leidy ; Cárdenas R. Ángela; García Obdulio \\ Universidad Nacional Abierta y a Distancia. Grupo de investigación
}

\section{Resumen}

El estudio está centrado en la identificación bromatológica del alpiste (Phalaris canarienses), verificando y constando sus niveles de concentración de grasa, fibra, macro y micro minerales, al igual su carga microbiana, confrontándola con los limites establecidos por la normatividad colombiana para categorizarlo como alimento apto para el consumo humano. Se realizo un proceso de elaboración de muestras liquidas del concentrado de la semilla de alpiste (Phalaris canariensis), que consiste en dejar en remojo 24 horas en agua destilada, luego al siguiente día lavarlo muy bien y proceder a licuarlo, filtrando las sustancia más gruesa para proceder a realizar las pruebas fisicoquímicas y microbiológicas;las pruebas se realizaron en el laboratorio de control de calidad de la Universidad de Pamplona cumpliendo con todas las normas de bioseguridad. Se realiza un análisis comparativo a nivel nutricional con la muestra de la semilla del alpiste frente al extracto del alpiste, determinando que los niveles de micro y macro minerales son más significativos en el grano que en el extracto, de la siguiente manera, en la semilla se encontraron mayores concentraciones de hierro grano $6,47 \mathrm{mg} / 100 \mathrm{gr}$ vs extracto $0.899 \mathrm{mg} / 100 \mathrm{gr}$ , magnesio grano $3,40 \mathrm{mg} / 100 \mathrm{gr}$ vs established by Colombian regulations to categorize it as food fit for human consumption. A process of preparation of liquid samples of the seed concentrate of canary seed (Phalaris canariensis) was carried out, which consists in leaving to soak 24 hours before in distilled water, then the next day to wash it well and to liquefy it, extracto $0.288 \mathrm{mg} / 100 \mathrm{gr}$, potasio grano $257,7 \mathrm{mg} / 100 \mathrm{gr}$ vs extracto $27.09 \mathrm{mg} / 100 \mathrm{gr}$ y zinc grano $1.75 \mathrm{mg} / 100 \mathrm{gr}$ vs extracto ND mg/100gr; y en la extracto de alpiste se encontró mayores concentraciones de magnesio y sodio, el magnesio en la extracto $12.9 \mathrm{mg} / 100 \mathrm{gr}$ vs grano $4.01 \mathrm{mg} / 100 \mathrm{gr}$ y el sodio en extracto $2.44 \mathrm{mg} / 100 \mathrm{gr}$ vs grano $0.46 \mathrm{mg} / 100 \mathrm{gr}$.

\section{Palabras clave:}

Alpiste, bromatológica, sustituto dietético, extracto, micronutrientes, macronutrientes.

\section{Abstract}

The study focuses on the bromatological identification of birdseed (Phalaris canarienses), verifying and recording its levels of concentration of fat, fiber, macro and micro minerals, as well as its microbial load, confronting it with the limits filtering the most thick to carry out the physicochemical and microbiological 
tests, the tests were carried out in the quality control laboratory of the University of Pamplona with all the biosafety regulations. In the results obtained, a comparative nutritional analysis is made with the sample of the seed of the birdseed compared to the birdseed extract, determining that the levels of micro and macro minerals are more significant in the grain than in the extract, of the following way, in the seed were found higher concentrations of iron grain $6.47 \mathrm{mg} / 100 \mathrm{gr}$ vs extract $0.899 \mathrm{mg} /$ $100 \mathrm{gr}$, magnesium grain $3.40 \mathrm{mg} / 100 \mathrm{gr}$ vs extract $0.288 \mathrm{mg} / 100 \mathrm{gr}$, potassium grain $257.7 \mathrm{mg} / 100 \mathrm{gr}$ vs extract $27.09 \mathrm{mg}$ / 100gr and zinc grain $1.75 \mathrm{mg} / 100 \mathrm{gr}$ vs extract ND mg / 100gr; and in the extract of canary seed was found higher concentrations of magnesium and sodium, magnesium in the extract $12.9 \mathrm{mg}$ / 100gr vs grain $4.01 \mathrm{mg} / 100 \mathrm{gr}$ and sodium in extract $2.44 \mathrm{mg} / 100 \mathrm{gr}$ vs grain $0.46 \mathrm{mg} / 100 \mathrm{gr}$.

Keywords: Birdseed, bromatological, dietary substitute, abstract, micronutrients, macronutrients.

\section{Introducción}

El consumo excesivo de alimentos con alto contenido calórico en la población colombiana ha provocado que miles de personas padezcan sobrepeso y obesidad; En Colombia la Encuesta Nacional de la Situación Nutricional (ENSIN, 2015) nos muestra el índice de sobrepeso y obesidad en personas adultas es $37,2 \%$ en mujeres y $38,3 \%$ en hombres. En el mundo $39 \%$ de las personas adultas de 18 o más años tienen sobrepeso, y el $13 \%$ son obesas. (OMS, Organización Mundial de la Salud, 2018) El sobrepeso y la obesidad son enfermedades metabólicas que se han expandido por todo el mundo, alcanzando
66

hoy una epidemia, representando el quinto factor de riesgo de mortalidad global.

Según Lau al menos 2,8 millones de adultos mueren cada año como resultado de tener sobrepeso, el $44 \%$ de los casos de diabetes, el $23 \%$ de casos de cardiopatía isquémica y entre $7 \%$ y $41 \%$ de ciertas cargas de cáncer son aplicables al exceso de peso y obesidad. (Lau, et al 2015) Acorde al estudio realizado por la OMS del 2016 menciona a nivel mundial más de 1900 millones tenían sobrepeso y más de 650 millones eran obesos. 2,8 millones mueren a cada año a causa de la obesidad. La presencia de personas obesas se triplicado en 1975 y 2016. Anteriormente era un problema que se reflejaba en los países de altos ingresos, actualmente es un problema que prevalece en los países de ingresos bajos y medianos se conoce que estas enfermedades están relacionadas con los hábitos alimenticios (Hannah y Howard, 1994; Wang et al., 2008) y que una alimentación saludable reduce el riesgo de sufrirlas (Sartori et al., 2009). Para llevar una vida sana es importante que nuestra alimentación contenga todos los elementos necesarios que el organismo demanda para su buen funcionamiento. Los productos suplemento dietético o funcionales representan una alternativa 12 para mejorar el estado de salud y en consecuencia la calidad de vida del ser humano (Afman y Muller, 2008); es el caso de los granos de soya, amaranto, alpiste y maíz (Vioque et al., 2000; SilvaSánchez et al., 2008). A partir de eso, las personas cada día están incluyendo en su alimentación alimentos más saludables y con propiedades hipolipidemica, indicando la reducción de 
concentraciones de lípidos en sangre entre lo que se puede citar al Alpiste (Phalaris canariensis) El alpiste (Phalaris canariensis) es una planta gramínea de la familia de las Poáceas. Es originaria del Mediterráneo, pero se cultiva comercialmente en varias partes del mundo para usar la semilla principalmente en la alimentación de pájaros domésticos, actualmente ha venido a despertar una gran cantidad de debates en lo que respecta a sus usos, siendo el más importante para el consumo humano. Al alpiste se le han otorgado un sin fin de beneficios para la salud de los seres humanos, por su alto valor nutricional, entre los más importantes el utilizado como desinflamatorio de los órganos. Sosulski (2001), demostró que la aplicación del alpiste posee una actividad antioxidante excelente, siendo sus principales componentes antioxidantes algunos esteroles, triterpenos y ésteres del ácido cafeico, por lo que convierte este dato al alpiste en un regenerador pancreático inmenso, así como una función desinflamante, favorecen a la diuresis y podría llegar a ser un incansable luchador contra el sobrepeso y la obesidad. Es una maravilla, por contener la enzima lipasa que elimina rápidamente grasa del organismo, ya sea de las arterias, o simplemente de los depósitos de grasa. (Line, 2005)

\section{Metodología}

Elaboración de la hidratación del alpiste

Figura. 1

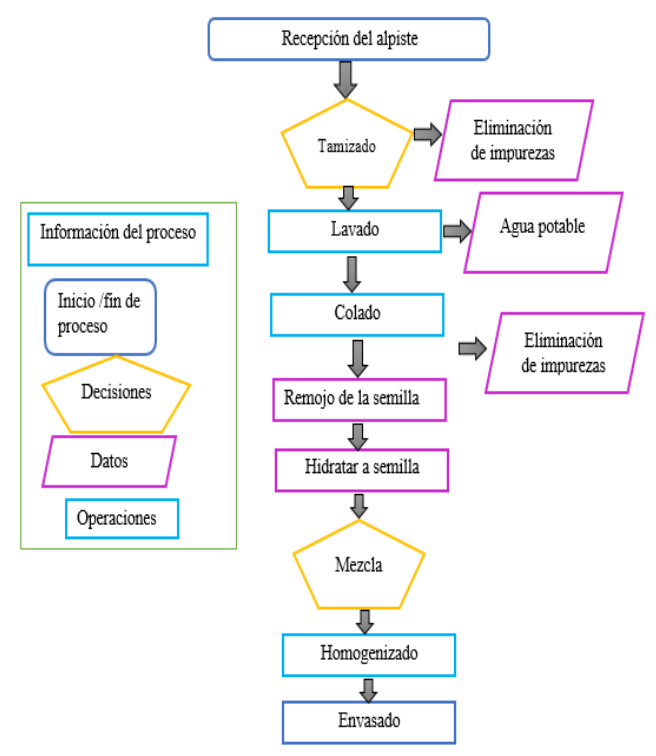

Fuente: Flujo grama de la elaboración de la extracto de alpiste natural. Fuente: elaboración propia

\section{Pruebas Fisicoquímicas de la Semilla de Alpiste y extracto}

\section{Determinación de humedad}

El contenido de agua de un producto se define convencionalmente como la pérdida de masa que experimenta en condiciones determinadas.

El producto se seca a 130 C bajo presión atmosférica normal durante 1,5 horas este método de desecación a 130 $\mathrm{C}$ aplica a los granos harinas y otros productos derivados de los cereales reducidos. (Madrid, 1994)

\section{Porcentaje de cenizas}

Se pesó $5 \mathrm{~g}$ de la muestra con aproximación de $\mathrm{mg}$ las restantes pesadas se hicieron de una aproximación de $0,1 \mathrm{mg}$.

Inmediatamente antes de usar las capsulas de incineración se calentaron en el horno a la temperatura de $910^{\circ} \mathrm{C}$ durante 15 minutos se enfriaron en el 
alcanzaron la temperatura ambiente.

Se Introdujo la muestra pesada en la capsula repartiéndola en una capa de espesor uniforme, sin comprimirla.

\section{Porcentaje de grasa}

Pese $5 \mathrm{~g}$ de la muestra molida de forma que pase por un tamiz de $500 \mu$ y desecada a $100^{\circ} \mathrm{C}$ e introducirlos en un cartucho que se tapona con algodón. Tarar el matraz, desecado en la estufa y enfriado en el desecador. Se introdujo el cartucho en el extractor, se añadió éter etílico una vez conectado el matraz se produjo a la extracción, continuándola hasta que el éter sea incoloro son suficiente 4 horas a una velocidad de destilación de 4 a 5 gotas y 16 horas para 2 a3 gotas. (America Associatión of ceral chesmist cereal, laboratorio de methods, 1967)

Se sacó el cartucho del extractor y recuperar el éter. Llevar el matraz con el extractor y el resto del disolvente a la estufa desecación a $100{ }^{\circ} \mathrm{C}$ y tenerlo media hora. Se dejó enfriar el matraz en el desecador y en cuanto alcance la temperatura ambiente pasearlo.

\section{Porcentaje de fibra cruda}

Pesé con aproximación de miligramos de 3 gramos de la muestra desengrasada y se secó. Coloqué en el matraz y adicioné $200 \mathrm{ml}$ de la solución de ácido sulfúrico en ebullición.

Coloqué el condensador y llevé a ebullición en un minuto; de ser necesario adiciónele antiespumante. Déjelo hervir exactamente por $30 \mathrm{~min}$, manteniendo constante el volumen con agua destilada y moviendo periódicamente el matraz para remover las partículas adheridas a las paredes.

\section{Tram}

Colocar los tubos estériles con sus tapones en la gradilla y adicionar a cada un $1 \mathrm{ml}$ de la solución de azul de metileno. Con la pipeta colocar $10 \mathrm{ml}$ de la muestra analizar y rotular.

Durante la preparación de las diferentes muestras, los tubos pueden mantenerse en baño maría de agua fría $\left(0-5^{\circ} \mathrm{C}\right)$ pero no más de dos horas.

Una vez preparados los tubos, taparlos e introducirlos en baño maría a $37^{\circ} \mathrm{C}$ junto con un tubo patrón (extracto sin indicador azul de metileno) (Figura 2). Cuando la temperatura de la muestra alcance $37^{\circ}$ mezclar el contenido de los tubos por inversión para obtener una perfecta homogeneización del colorante y el extracto. Tapar el baño maría para mantener los tubos al abrigo de la luz. (Frazier, 1992)

Pruebas de Absorción Atómica de los Metales Pesados Calcio, Magnesio, Hierro, Potasio, Sodio, Zinc, Manganeso en la semilla de Alpiste y extracto

\section{Espectroscopia de absorción atómica}

Se pesó una muestra de $10 \mathrm{~g}$ de grano entero de alpiste en un vaso Phillips de $250 \mathrm{ml}$. se agregó $40 \mathrm{ml}$ de $\mathrm{HNO} 3$ concentrado, cubra con vidrio y se dejó reposar a temperatura ambiente durante 2 horas. Se colocó el vaso de precipitados en un plato caliente y se caliento muy lentamente (reacción exotérmica). Después de que la reacción inicial cedió (35 minutos), calienta a $70{ }^{\circ} \mathrm{C}$ durante 3 horas. Se enfrió la solución y agregaron 2 $\mathrm{ml}$ de $\mathrm{HClO} 4$ al $70 \%$ y calentar hasta que se obtenga una solución clara. Cuando el proceso de ceniza se completó, $\mathrm{HClO} 4$ se 

trata a continuación con $5 \mathrm{ml}$ de $\mathrm{HCl}$ concentrado y el ácido se somete a reflujo en el vaso de precipitados. Se agregó un volumen igual de $\mathrm{H} 2 \mathrm{O}$ con evaporación posterior a sequedad. Este proceso se repito finalmente se añaden $1,0 \mathrm{ml}$ de $\mathrm{HCl}$ concentrado y la mezcla se calienta brevemente Se añaden $15 \mathrm{ml}$ de agua y se continúa calentando durante 15 minutos. Solución fría y transferir a un matraz volumétrico de $25 \mathrm{ml}$ y completar el volumen con H2O. (Perkin, 1994)

Se medió la concentración en espectrofotómetro de absorción atómica con el equipo shimadzu AA 7000

Lo que se va cambiando es la lámpara del mineral para calcio, magnesio, hierro, potasio, sodio, zinc, manganeso. (Perkin, 1994)

Pruebas Microbiológicas de la semilla de Alpiste y el extracto

\section{Aerobios mesófilos}

\section{Diluciones decimales}

Se transfirió con una pipeta $1 \mathrm{gr}$ (con una incertidumbre de medición de $\pm 5 \%$ ) de la suspensión inicial la semilla de alpiste en un tubo con $1 \mathrm{gr}$ del diluyente estéril. Para una óptima precisión no se introdujo la pipeta más de $1 \mathrm{~cm}$ en la suspensión inicial y se evitó el contacto entre la pipeta que contiene el inóculo y el diluyente estéril.

Se mezcló utilizando preferentemente un agitador mecánico durante 5 a 10 segundos para obtener la dilución 10-2. Se repitió esta operación a partir de la dilución 10-2

diluciones sucesivas, utilizando en cada operación una nueva pipeta estéril para obtener las siguientes diluciones 10-3. Se hizo las diluciones que sean necesarias para obtener un número apropiado de

microorganismos para realizar el recuento. (Fernando, 2014)

\section{Coliformes totales y fecales}

Para determinar la presencia de bacterias coliformes se utilizó la técnica del Número Más Probable (NMP), que consistió en realizar diluciones decuples seriadas, como se mencionó anteriormente, llegando hasta la dilución 10-3. Se depositó $1 \mathrm{ml}$ de cada dilución a cada uno de los tres tubos, conteniendo $11 \mathrm{ml}$ de caldo lactosado simple con tubo de Durham invertido para determinar la presencia de gas. Los tubos se incubaron durante 24 horas a $37^{\circ} \mathrm{C}$ y, transcurrido este tiempo, se revisaron los tubos que presentaron formación de gas, es decir, en los que se observó (Fernando, 2014))

\section{Mohos y levaduras}

\section{Preparación de la muestra}

$11 \mathrm{gr}$ de la semilla del alpiste de diluyente (dilución 1/10) y homogeneización (1 a 3 min).

Preparación de diluciones decimales: $1 \mathrm{ml}$ de la suspensión inicial $+9 \mathrm{ml}$ de semilla de alpiste del diluyente (dilución 10-2). Proceder de igual manera para las siguientes diluciones.

Inoculación e incubación: Sembrar una placa por dilución con $0.1 \mathrm{ml}$ de y $0.1 \mathrm{ml}$ de semilla de alpiste dilución decimal (10$2,10-3)$, en placas de Petri con Agar PDA Potato Dextrose Agar. Incubar a $25^{\circ} \mathrm{C} \pm$ $1 \stackrel{\circ}{ } \mathrm{C}$ durante 5 a 7 días. (Fernando, 2014))

\section{Resultados y análisis}

\section{Tabla 1.}


Resultados fisicoquímicos de la semilla de alpiste

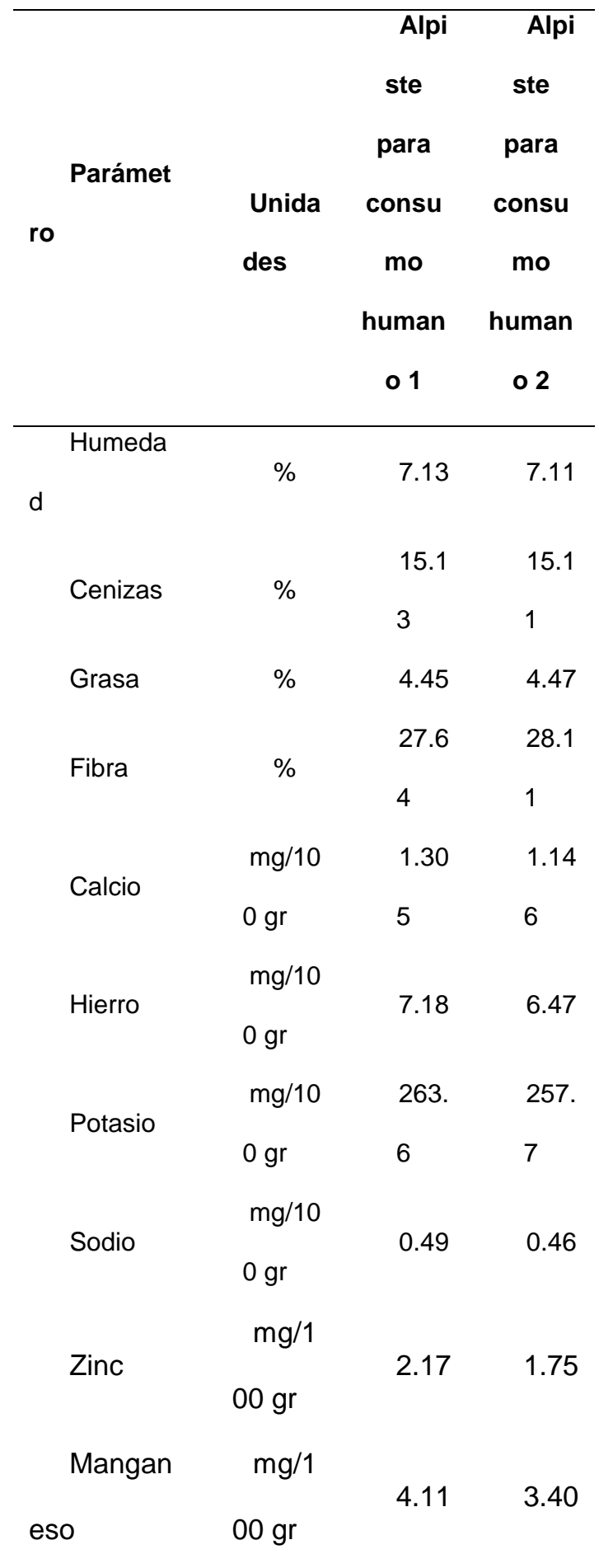

Fuente: Universidad de Pamplona, laboratorio de control de calidad y diagnóstico. Determinación de humedad
Según la tabla de composición de la semilla de apiste en 100 gramos comestible por Michel 2008, la semilla de alpiste posee un porcentaje de humedad $9.14 \%$, en las pruebas realizadas en el laboratorio a las dos muestras de semilla de alpiste para consumo humano arrojó un porcentaje de $7,13 \%$ y $7,11 \%$ de humedad, indicando que se encuentra entre rango medio comparado con la norma 529, el porcentaje de humedad para un grano dentro del rango es de $11.63 \%$ a $17.87 \%$, verificando que el contenido de humedad en un cereal es un índice de calidad. Es por ello que en base al contenido de agua se establecen las condiciones de manejo, transporte, almacenamiento y procesamiento de un cereal. (Charley, 2000) La absorción de agua puede ser especialmente problemática cuando el grano se almacena en condiciones de elevada humedad absoluta, tales como las que se dan en climas tropicales. (Culbertson y Grain, 2004)

\section{Porcentaje de cenizas}

El análisis de cenizas se obtuvo un resultado de $15,13 \%$ y $15,11 \%$, confrontándolo con la tabla de composición de la semilla de apiste en 100 gramos comestible por Michel 2008 donde referencia un porcentaje de cenizas en la semilla de alpiste de $13.48 \%$, se deduce con lo anterior que en la muestras analizadas cumple con los parámetros establecidos para cenizas, indicando que las muestras tiene un alto porcentaje de minerales. Según Matissek,. Et al 1992, define la ceniza como el residuo de incineración o el residuo que queda tras la combustión (incineración) completa de los 
composición de la semilla de alpiste en

componentes orgánicos de un alimento en unas condiciones determinadas. En dicho proceso de incineración se desnaturalizan los compuestos orgánicos, quedando el porcentaje de minerales, resistentes a las altas temperaturas.

\section{Porcentaje de grasa}

El análisis del porcentaje de grasa reporta un resultado en la semilla en cada una de las dos muestras analizadas de 4,45\% y un $4,47 \%$ confrontándola con la tabla de composición de la semilla de apiste en 100 gramos comestible por Michel 2008 con un parámetro de $5 \%$, indicando que la semilla de alpiste es una buena alternativa para reducir los niveles de colesterol del organismo, así como lograr controlar los niveles de azúcar en sangre (Buelvas,. et al, 2007)

\section{Porcentaje de fibra}

En el análisis de fibra se obtuvo un resultado de $27.64 \%$ y $28.11 \%$, confrontándolos con la tabla de composición de la semilla de alpiste en 100 gramos comestible por Michel, 2008 con un parámetro de $7,11 \%$, evidenciando que la muestra de alpiste en semilla tiene un índice superior al consultado, con lo anterior se valida sus propiedades como son la regulación de agua, reducción y absorción de lípidos y ácidos biliares regula el metabolismo de la glucosa efecto sobre la microbiota colonica (Mataix J, Gassull MA, 2002)

\section{Porcentaje de fibra}

En el análisis de fibra se obtuvo un resultado de $27.64 \%$ y $28.11 \%$, confrontándolos con la tabla de
100 gramos comestible por Michel, 2008 con un parámetro de $7,11 \%$, evidenciando que la muestra de alpiste en semilla tiene un índice superior al consultado, con lo anterior se valida sus propiedades como son la regulación de agua, reducción y absorción de lípidos y ácidos biliares regula el metabolismo de la glucosa efecto sobre la microbiota colonica (Mataix J, Gassull MA, 2002)

Tabla 2.

Resultados fisicoquímicos de la extracto de alpiste

\begin{tabular}{|c|c|c|}
\hline Parámetro & $\begin{array}{l}\text { Unidade } \\
\text { s }\end{array}$ & $\begin{array}{l}\text { Extracto } \\
\text { de alpiste } \\
\text { para } \\
\text { consumo } \\
\text { humano } 1\end{array}$ \\
\hline Tram & $\begin{array}{l}\text { Calidad } \\
\text { higiénica }\end{array}$ & $\begin{array}{c}\text { Inaceptabl } \\
\text { e }\end{array}$ \\
\hline Acidez & $\begin{array}{l}\text { \% Ácido } \\
\text { Láctico }\end{array}$ & 0.17 \\
\hline Calcio & $\mathrm{mg} / \mathrm{l}$ & 6.74 \\
\hline Magnesio & $\mathrm{mg} / \mathrm{l}$ & 12.9 \\
\hline Hierro & $\mathrm{mg} / \mathrm{l}$ & 0.899 \\
\hline Potasio & $\mathrm{mg} / \mathrm{l}$ & 27.09 \\
\hline Zinc & $\mathrm{mg} / \mathrm{l}$ & ND \\
\hline Sodio & $\mathrm{mg} / \mathrm{l}$ & 2.644 \\
\hline Manganes & & \\
\hline & $\mathrm{mg} / \mathrm{l}$ & 0.288 \\
\hline
\end{tabular}

Fuente: Universidad de Pamplona, laboratorio de Control de calidad y diagnóstico.

Prueba de Tram 
En análisis de tiempo de reducción del azul de metileno TRAM para el extracto de alpiste se obtuvo como resultado que la calidad higiénica es inaceptable debido a que desde la semilla existe un sin número de microorganismo por lo que no se lleva un proceso térmico entonces el potencial de óxido - reducción es muy elevado, el consumo de oxígeno será mayor y por consiguiente el óxido - reducción caerá rápidamente. (Ramos, 2003)

\section{Prueba de acidez}

Al analizar la acidez en el extracto de alpiste se obtuvo como resultado de 0.17 \% de ácido cítrico se encontró por encima del mínimo requerido para los néctares y refrescos de fruta $(0,2 \%$ de ácido cítrico anhidro (Ministerio de Protección Social y de Salud, Resolución 3929 de 2013, lo que indica que se acidifica rápidamente por lo cual hay que consumirlo de una manera inmediata puesto que los principales alteradores son los hogos estos pueden alterar la superficie del extracto como la semilla sin desinfección estos pueden ser fermentados rápidamente principalmente por la acción de levaduras alterando la calidad nutricional. (Ramirez, 1996)

\section{Pruebas de Minerales de la Semilla y el extracto de alpiste}

Frente a los resultados contenidos a partir de los análisis realizado a la semilla se encontró mayores concentraciones de hierro en el grano $6,47 \mathrm{mg} / 100 \mathrm{gr}$ vs extracto $0.899 \mathrm{mg} / 100 \mathrm{gr}$, magnesio grano $3,40 \mathrm{mg} / 100 \mathrm{gr}$ vs extracto $0.288 \mathrm{mg} / 100 \mathrm{gr}$, potasio grano 257,7 $\mathrm{mg} / 100 \mathrm{gr}$ vs extracto $27.09 \mathrm{mg} / 100 \mathrm{gr}$ y zinc grano $1.75 \mathrm{mg} / 100 \mathrm{gr}$ vs extracto ND mg/100gr; según Portela, (1993) los anteriores minerales son primordiales para el mantenimiento, estructural y funcional de la célula. En el extracto de alpiste se encontró mayores concentraciones de magnesio y sodio, el magnesio en la extracto $12.9 \mathrm{mg} / 100 \mathrm{gr}$ vs grano $4.01 \mathrm{mg} / 100 \mathrm{gr}$ y el sodio en
72

extracto $2.44 \mathrm{mg} / 100 \mathrm{gr}$ vs grano $0.46 \mathrm{mg} / 100 \mathrm{gr}$, confrontando los resultados con la resolución 3803 del ministerio de salud y protección social, indica el índice recomendado de macro y micro minerales para la población colombiana en general de la siguiente manera: calcio 700-1.300 mg/día magnesio 80-420 mg/día sodio 120-1.500 $\mathrm{mg} /$ día y potasio $400-5100 \mathrm{mg} /$ día y en los micro minerales hierro 11-27 mg/día zinc 3-14 mg/día. Nutrientes como el sodio el importante para el hombre ayudando a controlar la regulación de la osmolaridad 0 presión osmótica (diferencia de concentración a nivel de membrana celular), y control del balance o equilibrio acido básico metabólico; el magnesio a intervenir en la transmisión del impulso nervioso y en la relajación muscular, el calcio necesario para el mantenimiento del equilibrio ácido-base, interviene en las acciones de la parathormona (hormona que interviene en la regulación del metabolismo del calcio y del fósforo), y la vitamina $D$ del hueso. (Angeles A. , 2013)

Tabla 3

Resultados microbiológicos de la semilla y del extracto de alpiste

\begin{tabular}{crc}
\hline Parámetro & $\begin{array}{r}\text { Alpiste } \\
\text { en } \\
\text { semilla } \\
\text { consumo } \\
\text { humano }\end{array}$ & $\begin{array}{c}\text { Extracto } \\
\text { alpiste } \\
\text { consumo } \\
\text { humano }\end{array}$ \\
\hline Aerobios & $52 \times$ & $92 \times 10^{6}$ \\
mesófilos & $10^{4} \mathrm{ufc} / \mathrm{g}$ & ufc/g \\
Coliformes & $27 \times$ & $43 \times 10^{3}$ \\
totales & $10^{2} \mathrm{ufc} / \mathrm{g}$ & $\mathrm{ufc} / \mathrm{g}$ \\
Coliformes & $7 \times$ & $21 \times 10^{2}$ \\
fecales & $10^{2} \mathrm{ufc} / \mathrm{g}$ & ufc/g \\
\hline
\end{tabular}


determinó un recuento $43 \times 103$ el cual sobrepasa los límites establecidos por la resolución ministerial nํ 363-2005/MINSA estableciendo un rango de $102 \times 103$; con los resultados anteriores y frente a la normatividad se establece que las muestras analizadas no son NO APTAS para el consumo humano. Estos microrganismos establecen un grupo bacteriano heterogéneo, con especies de origen intestinal y no intestinal. Un recuento alto de UFC de coliformes totales indica que el jugo o extracto de alpiste estuvieron bajo condiciones que los pueden convertir en un peligro para la salud. (Borbolla,M., et al 2004)

\section{Coliformes fecales}

El análisis microbiológicos de Coliformes fecales en la semilla de alpiste, determinó que los recuentos exceden los límites determinados por la normatividad colombiana de la resolución ministerial 201 del 2012 del INVIMA el cual establece la no presencia de UFC $/ \mathrm{ml}$ frente a $7 \mathrm{X}$ $10^{2} \mathrm{ufc} / \mathrm{ml}$ por lo tanto se toma esta muestra como NO APTA para el consumo humano. Con el extracto se determinó un recuento de 21×102 el cual sobrepasa los límites establecido por la resolución ministerial no 363-2005/MINSA que determina un rango de $10-102$, con la confrontación de los resultados obtenidos en la muestra del extracto con la norma se establece que NO es APTA para el consumo humano.

Frente a la importancia de los Coliformes totales como indicadores de contaminación del agua y de los alimentos y a pesar que la normatividad colombiana de la resolución ministerial 201 del 2012 del INVIMA no lo exigen para los granos, se decidió realizar de manera opcional el análisis microbiológico de Coliformes totales a la muestra de semilla, con un recuento de UFC de $27 \times$ $10^{2}$, revelando la presencia de bacterias de este género en la muestra $y$ evidenciando la contaminación de la misma. En el caso del extracto se

La prueba de coliformes fecales positiva indica un $90 \%$ de posibilidad de que el coliforme aislado sea Escherichia coli. Los coliformes fecales son un indicador de contaminación fecal en alimentos y por tanto establece la calidad del alimento. (Prado., et al 2013)

\section{Mohos y levaduras}

El análisis microbiológicos de Mohos y levaduras en la semilla de alpiste, estableció que los recuentos sobrepasa las especificaciones microbiológicas 
determinados por la normatividad colombiana de la resolución ministerial 201 del 2012 del INVIMA el cual establece un máximo 50000 $\mathrm{ufc} / \mathrm{ml}$ frente a $5700 \mathrm{ufc} / \mathrm{ml}$ en el análisis de la semilla, por lo tanto se toma esta muestra como NO APTA para el consumo humano.

Frente a la importancia de los mohos y las levaduras como microorganismos indicadores que determinan la calidad sanitaria de alimentos y a pesar que la normatividad colombiana de la resolución ministerial $n^{\circ}$ 363-2005/MINSA no lo exigen para los jugos no procesados térmicamente que es el caso del extracto de alpiste, se estableció realizar de manera opcional el análisis microbiológico de mohos y levaduras, el cual se obtuvo un recuento de 7100 UFC, revelando la presencia de bacterias de este género en la muestra y demostrando la contaminación de la muestra y estableciendo que la muestra no es APTA para el consumo humano, puesto que se encuentran ampliamente distribuidos en el ambiente, por lo que son frecuentes en la microbiota habitual de muchos alimentos; se dispersan fácilmente por el aire y el polvo. (Pirson, y Smoot, 2001)

\section{Conclusión}

A nivel nutricional que la concentración de micro y macro minerales es mayor en la semilla que en el extracto de alpiste, perdiendo parte de sus nutrientes atrapados en el bagazo. Con respecto a la concentración de fibra obtenida en el análisis bromatológico se obtuvo un resultado de $28.11 \% / 100 \mathrm{gr}$ frente la tabla de composición de la semilla de alpiste en 100 gramos comestible de Michel, 2008 de $7.11 \%$, con los siguientes resultados se verifico y ratifico la importancia que tiene el alpiste como alternativa para el tratamiento del sobre peso y la obesidad, gracias a la grandes concentraciones de fibra alimentaria significativa para las funciones fisiológicas, ya que ella ayuda a movilizar los alimentos a través del aparato digestivo hasta el duodeno, así como la orina desde los riñones a la vejiga, procesos llamados perístasis intestinal, es decir que con una dieta rica en fibra alimentaria, característica relevante del alpiste, para el tratamiento de enfermedades renales, hipertensión, hiperglucemia, hipercolesterolemia, sobrepeso y obesidad (Cogliatti, 2012)

\section{Referencias Bibliografícas}

74

America Associatión of ceral chesmist cereal, laboratorio de methods. (1967).

Angeles, A. (2013). Manual de nutrción. Madrid.

Buelvas, H., Arrieta, , G., Arrázola , P., \& Martínez, C. (2007). Análisis fisicoquímico y agronómico de frutos y semillas de almendro de la India (Terminalia catappa L.). Fruticultura Profesional, 37-44.

Borbolla, M., Perez, O., Piña , , I., Vidal, , J., \& Vidal, , M. (2004). Contaminacion de los alimentos por vibrio cholera, coliformes totales, salmonella y hogos y levaduras. Villa Hermosa Mexico.

Culbertson, J., \& Grain, D. (2004). Cereal: Ready-to-Eat Breakfast Cereals, en Food Processing: Principles and Applications, J. Scott Smith y Y. H. Hui . Blackwell Publishing, 34-35.

ENSIN. (2015). Encuesta Nacional de situacion Nutriconal. Colombia: Instituto Colombiano de Bienestar Familiar.

Fernando, T. (2014). Analisis microbiologicos delos alimentos. Trelew - Chubut: INAL ANMAT.

Frazier, W. (1992). Metodos de laboratorio en microbiologoa de alimentos y productos lacteos. En W. Frazier, Microbiologia de alimentos 4 edición . Zaragoza: Acribia.

Hernández Garcia Jessika Andrea ., Ariza Garcia Jherson, Cano Calle Herminsul de Jesús,Contreras Pineda Jorge .2013. Estandarización de una técnica para la certificación de jardines clonales de plantas de cacao(Theobroma cacao ) usando marcadores moleculares rapd. Bistua:Revista de la Facultad de Ciencias Básicas.10(2):75-84

Lau, E., Carvalho, D., Pina-Vaz, C., Barbosa, J., \& Freitas, P. (2015). Beyond gut microbiota: understanding obesity and type 2 diabetes. hormones (athens), 358 -369.

Madrid, V. (1994). Metodo oficial de analisis de los alimentos. España: Mundi prensa.

OMS. (10 de Marzo de 2018). Organización Mundial de la Salud. Obtenido de 
http://www.who.int/es/news-room/factsheets/detail/obesity-and-overweight

Perkin, E. (1994). Atomic absorption spectrocopy analytical methods. Madrid vicente : Ediciones.

Portela, M. (1993). vitaminas y minerales en nutrción . Colombia: Libreros Lopez Editores.

Prado, A. R. (2013). Manual de prácticas de laboratorio de microbiologia de los alimentos Universida Autonoma Metropolitana. Mexico.

Pirson, M, \& Smoot, , L. (2001). Food Microbiology. Fundamentals and Frontiers. 2nd ed. Doyle M. En P. M, \& L. Smoot, Indicator Microorganisms and Microbiological (págs. 71-87). USA: ASM Press.

Ramos, E. (2003). Introducción al control de calidad de la leche cruda.catedra de ciencias y tecnologia de leche. revistra virtual pro, ww.revistavirtualpro.com/files/ti20_200512.pd f.

Yaguez, J. L. (23 de marzo de 2002). Inta. Obtenido de Alpiste un cultivo olvidado: WWW.INTA.GOV.AR/BARROW/INFO

*Para citar este artículo: Peña C. L.; Cárdenas R. Á.; García O. Bromatological analysis of milk from canary seed (phalaris canarienses): cereal used as a dietary substitute. Revista Bistua. 2019 17(1):6575

+ Autor para el envió de correspondencia y la solicitud de las separatas: Cárdenas R. Ángela Universidad Nacional Abierta y a Distancia.

Recibido: Marzo15 de 2018

Aceptado: Agosto 10 de 2018 